\title{
DIFFERENT TYPES OF PHOTOMETRIC AND POLARIMETRIC BEHAVIOUR OF AM HER IN 1989-1994
}

\author{
N. M. SHAKHOVSKOY ${ }^{1}$, I. L. ANDRONOV ${ }^{2}$, S. V. KOLESNIKOV ${ }^{2}$ \\ 1. Crimean Astrophysical Observatory \\ 334413 Nauchny, Crimea, Ukraine \\ 2. Department of Astronomy, Odessa State University \\ T.G. Shevchenko Park, 270014 Odessa, Ukraine
}

Abstract. Variations in $U B V R I$ are classified as: luminosity- and wavelength- dependent phase variations with differences from cycle-to-cycle; flares caused by accretion events; UV Cet-type flares from the secondary.

More than 60 nights of observations in various luminosity states allow us to distinguish the following types of photometric activity.

(i) Regular variations with the orbital phase in $R$ and $I$, which are less pronounced in $V$ and practically invisible in $B$ and $U$.

(ii) Irregular flares with different time-scales. Usually they are best seen in $R$ and $I$, but sometimes 'white' flares occurred with similar amplitudes in all bands. Both types of variability are observed mainly in the bright state of the star and are due to cyclotron emission in the accretion column(s).

(iii) Smooth variations in $U$ and $B$ (at the 1st harmonic of the orbital period). These are often opposite to variations in $R$ and $I$. They are most prominent in the 'intermediate' and 'low' states and may be explained by the inhomogeneity of the surface brightness of the red component (reflection effect or spot).

(iv) Fast 'blue' flares, most prominent in $U$ and $B$. These were similar to a giant flare which occurred on 1992 August 29 and are probably arising on the red dwarf. They resemble the flares of UV Cet-type stars.

\section{References}

1. Andronov, I.L., Kolesnikov, S.V., Shakhovskoy, N.M., 1992, in "Stellar Magnetism", ed. Yu.V. Glagolevsky, (S.-Peterburg), p148

2. Shakhovskoy, N.M., Alexeev, I.Yu., Andronov, I.L., Kolesnikov, S.V., 1993, in "Cataclysmic Variables and Related Physics", eds O. Regev, G. Shaviv, Ann. Israel Phys. Soc., 10, 237

A. Evans and J. H. Wood (eds.), Cataclysmic Variables and Related Objects, 209-210. () 1996 Kluwer Academic Publishers. Printed in the Netherlands. 


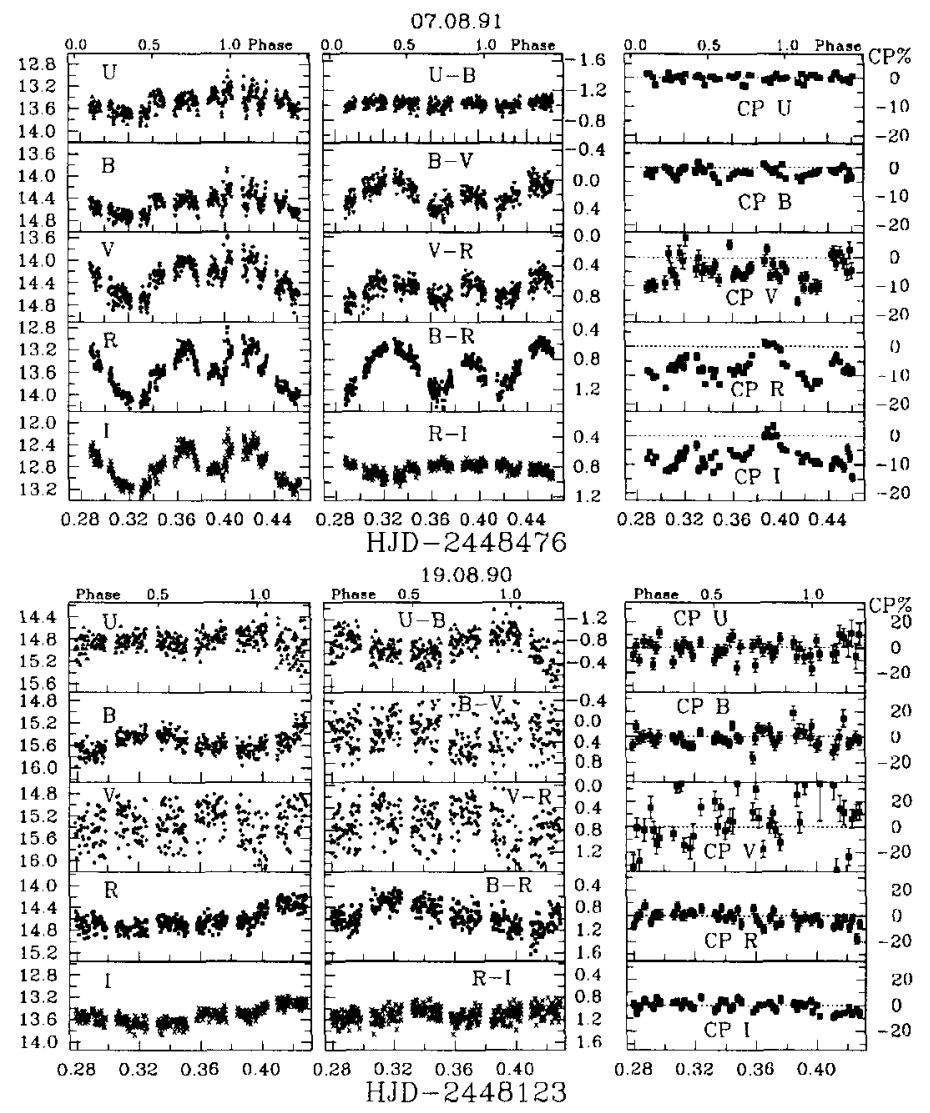

Figure 1. UBVRI brightness, color and circular polarization variations of AM Her in the high (top; 1991 August 7, JD 2448 476) and low (bottom; 1990 August 19, JD 2448 123) luminosity states. Bottom abscissae show the decimal part of HJD, upper ones, the polarimetric phase. The flares in the light curves (top) are not seen in all colours. During long states of low activity, the circular and linear polarization and the fast variability sometimes completely disappear. At these times AM Her loses all characteristics of the type and formally can not be classified as a 'polar' [1]. The unprecedented UV Cet-type flare on 1992 August 29 was also observed during the low state [2]. 